Service social

\title{
l'analyse des mouvements sociaux urbains : questions de méthode
}

\section{Lionel Groulx}

Volume 34, numéro 2-3, 1985

L'organisation communautaire

URI : https://id.erudit.org/iderudit/706274ar

DOI : https://doi.org/10.7202/706274ar

Aller au sommaire du numéro

Éditeur(s)

École de service social de l'Université Laval

ISSN

1708-1734 (numérique)

Découvrir la revue

Citer cet article

Groulx, L. (1985). l'analyse des mouvements sociaux urbains : questions de méthode. Service social, 34(2-3), 300-307. https://doi.org/10.7202/706274ar d'utilisation que vous pouvez consulter en ligne.

https://apropos.erudit.org/fr/usagers/politique-dutilisation/ 
Groulx, Lionel, professeur à l'École de service social de l'Université de Montréal.

\section{L'analyse des mouvements sociaux urbains : questions de méthode}

\section{Lionel Groulx}

Les mouvements sociaux urbains sont l'objet et l'enjeu de plusieurs interprétations qui renvoient souvent à des visions différentes du social. Ces divergences ou controverses sont parfois interprétées comme des retraductions de positions idéologiques ou politiques sans que soient toujours examinés les fondements épistémologiques et méthodologiques de ces débats. Nous voudrions, dans cet article, approfondir ces deux dimensions selon lesquelles le rapport à l'objet détermine en partie le discours sur celui-ci. Dans l'étude des mouvements sociaux urbains, ce rapport s'établit selon deux modalités : la participation ou l'observation, double posture gnoséologique qui oriente analyses et interprétations.

Après avoir présenté cette double logique de construction de l'objet, nous illustrerons l'antinomie de ces deux modes de connaissance par la comparaison de la double analyse de $\mathrm{McGraw}^{1}$ et de Collin-Godbout ${ }^{2}$ sur les mouvements sociaux urbains.

\section{Dilemmes entre l'observation et la participation}

Chacune des logiques, soit d'observation soit de participation, véhicule un rapport à l'objet d'étude différent. L'une insiste sur la distance, sur la position d'extériorité, et l'autre, sur la participation à l'objet et sur sa familiarisation progressive ; cela se traduit, dans chaque cas, par des normes différentes d'appartenance et de référence, dont l'une est constituée par la tradition disciplinaire avec une visée davantage explicative, et l'autre par le projet social du mouvement avec une exigence plus compréhensive. Chaque position produit une définition opposée du chercheur, dans un cas comme objectif ou neutre, dans l'autre comme militant ou engagé. 
La logique de la participation pousse à une intériorisation progressive des normes du mouvement et ce, jusqu'à l'engagement militant qui amène à privilégier son autonomie et à développer ses capacités virtuelles. Ceci amène à véhiculer une interprétation davantage volontariste ou indéterministe de l'action sociale produisant, comme l'exige toute participation intense à un phénomène, un effet d'agrandissement, ${ }^{3}$ qui accentue ou exagère les possibilités virtuelles et spécifiques de changement social. Cette tendance est renforcée par l'interaction quotidienne avec les militants eux-mêmes qui demandent au chercheur-participant de se joindre à la construction symbolique de l'identité du mouvement lui-même. Cette insistance sur une vérité plus compréhensive ou, du moins, plus phénoménologique, axée sur l'expérience vécue de l'action, se différencie fortement de la logique de l'observation, centrée sur la nécessité d'objectivation par une distanciation ou une extériorité. Dans ce type de rapport, les questions principales renvoient davantage au paradigme d'interprétation de l'observateur. Ces nouvelles interrogations, à partir de catégories extérieures, parfois étrangères au mouvement, portent à ne pas intérioriser ou faire siennes les significations qu'il produit mais plutôt à les décontextualiser ou, du moins, à critiquer et soupçonner sa vérité subjective ou immédiate. Cette dernière démarche intellectuelle (observation) prédispose à une perception plus déterministe, voire mécanique de l'action sociale et, dans certains cas, réductionniste ou désenchantée dans la contestation objectiviste de la vérité spontanée que les membres militants donnent de leur action. Pour la logique de l'observation et ce, dans presque tous les cas, le mouvement est renvoyé à autre chose que lui-même, ou conçu comme variable dépendant de processus psychosociaux plus complexes. Cette perspective l'amène à remettre en question son autonomie et ses capacités potentielles de transformations sociales.

\section{La double analyse de McGraw et Collin-Godbout}

La double lecture de McGraw et de Collin-Godbout sur les groupes populaires illustre cette difficulté d'une connaissance scientifique des mouvements sociaux. Construites à partir de perspectives différentes, ces deux recherches aboutissent à des résultats différents sinon contradictoires. Ces divergences proviennent en partie d'un rapport différent à l'objet, i.e. du statut de participant-militant chez McGraw et d'observateur extérieur chez Collin-Godbout.

McGraw, ancien animateur au Conseil de développement social, a été, selon ses propres termes, "lié aux luttes en milieu urbain montréalais à certains moments de leur développement $» .^{4} \mathrm{Ce}$ statut de 
participant-militant l'amène à concevoir les groupes populaires en termes de mouvement social et à développer un cadre d'analyse militant, d'orientation marxiste de type gramscien qui donne une importance stratégique et analytique aux intellectuels-animateurs des groupes populaires.

"Nos développements théoriques nous amèneraient à chercher l'origine des mouvements de lutte dans le développement progressif d'une nouvelle pratique de direction hégémonique de l'État à travers le rôle charnière d'intellectuels organiques. ${ }^{5}$

Cette insistance sur les intellectuels-animateurs-militants est en harmonie avec une conception volontariste de l'action sociale où l'évolution des mouvements de luttes est réductible à la volonté des initiateurs, i.e. des intellectuels.

"Nous croyons que les mouvements de lutte en milieu urbain ne progressent pas vers une rupture selon une logique qui leur serait propre : ils évoluent selon une logique qui est inspirée des leçons que tirent, de leur pratique, les initiateurs de ces mouvements de lutte. ${ }^{6}$

Ce qui amène à privilégier, comme matériel empirique et comme données de preuve, le discours, i.e. l'analyse explicite des agentsintellectuels.

"Ces organisations ont été initiées par des individus qui développaient une analyse des causes des problèmes sociaux et qui élaborèrent des objectifs, stratégies et moyens conséquents, dont les organisations sont une expression. » ${ }^{7}$

Cette analyse provient, en partie, du rapport de participation, qui incline à une intériorisation progressive des normes du mouvement, ce qui amène à privilégier l'autonomie de ce dernier, ses capacités virtuelles en véhiculant une interprétation davantage indéterministe de l'action sociale. Ceci produit, comme l'exige toute participation intense à un phénomène, un effet d'agrandissement qui accentue ou exagère les possibilités virtuelles et spécifiques de changement social. L'analyse participe alors à la construction symbolique de l'identité du mouvement populaire comme telle.

Elle se différencie fortement de celle menée par Collin-Godbout, qui travaillent comme chercheurs à l'I.N.R.S.-Urbanisation. Les interrogations qu'ils véhiculent s'établissent à partir de catégories extérieures, voire étrangères aux groupes eux-mêmes. Ils vont même jusqu'à faire leur la sociologie spontanée des "individus en place".

"Bref les comités de citoyens, est-ce une affaire d'animateurs? Ou sont-ils devenus autonomes? C'est l'argument avancé par les institutions et les individus en place lorsqu'ils veulent nier la représentativité 
et donc la pertinence des organismes populaires. Est-ce une raison suffisante pour rejeter l'hypothèse ? 8

Les auteurs récusent cette vision à partir du discours des militants ou des significations véhiculées par les agents eux-mêmes.

"Cette approche, différente et impliquant le traitement d'un ensemble d'informations factuelles, se situe à un autre niveau que l'analyse du discours. ${ }^{9}$

Ce qui amène à privilégier comme éléments de preuve des données quantifiables et mesurables selon une conception davantage positive des faits sociaux.

"[...] de la connaissance la plus systématique et la plus complète possible de ce qu'ils font, de ce qu'ils produisent, de leur output (service, bien, dans quel secteur, etc.), de la façon dont ils le produisent (structure de l'organisme, rôle des différents agents, etc.) et des ressources qu'ils utilisent pour produire (finance, source, relation avec l'État, avec d'autres organismes, etc.) [...]"10

Cette démarche favorise une conception quasi mécanique de l'action sociale. ${ }^{11}$ Les auteurs s'intéressent moins à la dynamique de l'action sociale comme telle qu'aux variables qui déterminent et structurent l'action des groupes populaires. Contrairement à la perspective de McGraw, cette analyse extérieure et "observatrice" produit un effet de réduction dans le jugement porté sur les agents-animateurs eux-mêmes et sur les groupes populaires comme tels. Dit autrement, la perspective externe, extérieure et observatrice, rejette la signification véhiculée par les acteurs, privilégie les activités en elles-mêmes et produit un désenchantement, tant de l'initiative des animateurs que de la volonté politique des groupes eux-mêmes.

Cet effet de réduction se révèle dans la contestation que CollinGodbout font de la vérité subjective et spontanée que les militants donnent de leur action. Comparativement à McGraw, qui considère les animateurs sociaux comme initiateurs des luttes sociales et relie les stratégies d'action à leur analyse de la situation et de l'état des rapports politiques avec le pouvoir municipal, Collin-Godbout renvoient les stratégies d'intervention des animateurs au problème de leur propre légitimation. Leurs stratégies d'action ne dépendent plus de leur perception de l'état de la lutte sociale mais de la défense de leurs propres intérêts.

"Cet effritement de leur leadership est une des causes majeures qui obligent les animateurs à réviser périodiquement leurs stratégies d'intervention dans les quartiers et dans les organisations populaires. ${ }^{12}$ 
Cette interprétation remet aussi en question la signification politique de l'action des groupes populaires.

"[...] La faiblesse de l'hypothèse voulant que ces groupes constituent la base d'un contre-pouvoir dans les quartiers et la présence importante des animateurs à chaque niveau de la vie politique des groupes de citoyens. ${ }^{13}$

"[...]la participation (ou non) des groupes populaires aux élections municipales apparait comme l'affaire des animateurs - en tout cas apparaît plus l'affaire des animateurs que celle des citoyens. „14

"En l'absence (ou presque) de cette dimension politique - que tentent, en vain jusqu'à maintenant, de leur insuffler certains militants $[\ldots]{ }^{15}$

Étant une "affaire d'animateurs", la revendication politique des groupes populaires devient, pour ceux-ci, et dans ce raisonnement, un phénomène extérieur et étranger. On aboutit alors à les concevoir, non plus comme un mouvement de lutte, mais comme des groupes de pression.

"Les organismes populaires ne sont pas un contre-pouvoir [...] nouveau mécanisme d'exercice du pouvoir et de distribution des biens, et non pas volonté de redistribution du pouvoir politique, ce que serait un mouvement politique. " 16

"Les groupes agissent essentiellement comme des groupes de pression et ils ne procèdent pas à partir d'une philosophie politique exprimée ou d'un programme général mais plutôt à partir de contre-projets à des projets publics ou privés. 17

Au terme de ce raisonnement, peut-on espérer une connaissance scientifique des mouvements sociaux? La comparaison des deux recherches précitées rend plutôt sceptique. Ces deux analyses ne font que retraduire et systématiser la visée spontanée que chaque protagoniste a de son action. Rôle initiateur des intellectuels et caractère de mouvement social pour une perspective participante, et rôle manipulateur des intellectuels et caractère de groupes de pression pour une perspective observatrice. Tout se passe comme si chacun des auteurs "importait dans l'objet les principes de leur relation à l'objet». ${ }^{18}$ Posture de participant ou d'observateur, qui favorise des conceptions différentes de l'action sociale et prédispose à des interprétations contradictoires du phénomène, ce qui produit alternativement des effets d'agrandissement ou de réduction. À la limite, la relation de recherche est spéculative, car chacun des auteurs confirme l'articulation de ses propositions initiales. 


\section{TABLEAU 1}

La double lecture de la mobilisation sociale à partir de la comparaison entre McGraw et Collin-Godbout

\begin{tabular}{|l|l|l|}
\hline \multicolumn{1}{|c|}{ Variable } & \multicolumn{1}{c|}{ McGraw } & \multicolumn{1}{c|}{ Collin-Godbout } \\
\hline Rapport à l'objet & $\begin{array}{l}\text { Participant-militant } \\
\text { Interne }\end{array}$ & $\begin{array}{l}\text { Observateur extérieur } \\
\text { Externe }\end{array}$ \\
\hline Sources de données & Discours des animateurs & Activités des groupes \\
\hline Conception de l'action sociale & Volontariste & Déterministe \\
\hline Conception des groupes populaires & Mouvement social & Groupes de pression \\
\hline Perception des animateurs sociaux & Initiateur & Manipulateur \\
\hline Cadre d'interprétation & $\begin{array}{l}\text { Herméneutique } \\
\text { Compréhension }\end{array}$ & $\begin{array}{l}\text { Positivisme } \\
\text { Explication }\end{array}$ \\
\hline Effet & Agrandissement & Réduction \\
\hline
\end{tabular}




\section{Conclusion}

On pourrait objecter que la sortie de ce dilemme pourrait se faire par l'observation participante, "sorte d'expérience croisée du monde social, à savoir la familiarisation avec un monde étranger et le déracinement d'un monde familier ". ${ }^{19}$ Mais la pratique concrète de ce type d'observation nous renvoie aux contradictions pratiques qu'impose son utilisation, car elle commande l'intégration et la gestion des statuts d'observateur extérieur et de participant militant, posture qui favorise une double construction symbolique et sociale de la réalité et une double expérience contradictoire, sinon incompatible, du monde social, ce qui rend difficile une connaissance scientifique des mouvements sociaux urbains. On comprend mieux, à cet égard, que les analyses, dans la plupart des recherches sur ceux-ci, tendent presque automatiquement, par une série de choix infinitésimaux et successifs, à confirmer l'articulation des propositions initiales car, aux divers moments de la recherche, celles-ci deviennent le seul principe cohérent de lecture et d'interprétation. Ce " cercle vicieux méthodologique" reste trop souvent nié ou occulté dans les analyses sur les mouvements sociaux urbains, plus intéressés à affirmer le sens des actions et des mobilisations sociales qu'à définir les limites de validité de leurs interprétations. ${ }^{20}$

\section{Notes et références}

1 McGraw, D., Le développement des groupes populaires à Montréal (19631973), Montréal, Éditions Saint-Martin, 1978, p. 13.

2 Collin, J.-P. et J. Godbout, Les organismes populaires en milieu urbain, Montréal, I.N.R.S.-Urbanisation, 1 re édition, 1975, p. 7.

3 Comme le note Merton qui reprend, à sa manière, le dilemme de l'observation et de la participation, cette situation peut mener, à la limite, à une sorte de solipsisme méthodologique, à une balkanisation de la connaissance du social où le privilège de cette connaissance est réductible à sa participation. (R.K. MERTON, The Sociology of Science, Chicago, University of Chicago Press, 1973, pp. 99-138.)

4 McGraw, D., op. cit.

5 Id., p. 28.

6 Id. , p. 37.

7 Id. , p. 53.

${ }^{8}$ Collin, J.-P. et J. Godbout, op. cit.

9 Id., p. 6. 
10 Id. , p. 5.

11 Id., p. 100.

12 Id., p. 51.

13 Id. , p. 209.

14 Id., p. 211.

15 ld. , p. 227.

16 lbid.

17 Id. , p. 89.

18 Bourdieu, P., Esquisse d'une théorie de la pratique, Paris, Droz, 1972, p. 160. L. Groulx, "Action sociale et lutte populaire", Cahiers canadiens de sociologie, vol. 6, nº4, 1982 : 463-484.

19 Bourdieu, P., ibid.

20 II est à remarquer que ce couple épistémologique et méthodologique (participation et observation) est irréductible aux couples subjectif-objectif ou quantitatif-qualitatif et qu'il ne se réduit pas à la seule analyse des mouvements sociaux. 